Recibido: 11-07-2016

Aceptado: 26-07-2016

Palabras clave:

Neuropsicologia, sexualidad, Comportamiento, educación, adolecencia.

Keywords:

Neuropsichology, sexuality, behavior, education, adolescense.

carmen.montenegro@upch.pe

UPCH

Geraldine Salazar Vargas geraldine.salazar@upch.pe UPCH

Hugo Salazar Jáuregui hugo.salazar@upch.pe UPCH

Luis Aguilar UPCH - UNIFE luis.aguilar@upch.pe

\section{Conocimientos y actitudes sobre anticoncepción en estudiantes de 5 to. año de secundaria de la ciudad del Cusco}

\section{Knowledge and attitudes about contraception in high school's 5 th grade students of the city of Cusco}

\author{
Estela Montenegro Serkovic', Geraldine Salazar Vargas', Hugo \\ Salazar Jáuregui', Luis Aguilar ${ }^{1.2}$
}

RESUMEN. La presente investigación es explorativa, descriptiva, transversal. El estudio se realizó con hombres y mujeres de 5to Secundaria en un colegio del Cusco. El objetivo fue identificar actitudes y conducta sexual afectiva en referencia a la contraceptación.

$77 \%$ de los estudiantes vivian con sus padres. $37 \%$ de ellos tenían educación superior completa y $26 \%$ con educación secundaria completa. Se halló que la familia es la princiapl fuente de educación sexual en referencia a la contracepción, la madre es la que mayormente brinda información al sujeto.

Asiismo los diarios como medio de comunicación social preferido. Se encontró que prevalecen actitudes semi liberales.

Se recomendó el desarrollo de una estrategia centrada en la enseñanza y aprendizaje de familia.

ABSTRACT. The present reseaech exploratory, descriptive, transversal. Study with men and womwn of the 5th Secondary of Cusco. The objetive was to identify attitudes ans sexual affective behavior in reference to contraception. $77 \%$ of the students live with their parents. $37 \%$ with complete higher education and $26 \%$ with secondary education. The family is the main source of sexual information i reference to contraception. The mother is the one who infors on the subject.

Newspapers rank first among the media. It was found that precailing semiliberal attitudes.

It is recommended to develop a strategy of family-centered cognitive behavioral teaching and learning. 


\section{Introducción}

$\mathrm{L}$

a iniciación sexual es un evento que normalmente ocurre durante los últimos años de la adolescencia. Si la práctica sexual se da en forma precoz, sin la planificación ni la protección necesaria trae consecuencias indeseadas para el individuo, se convierte en un problema central para él y puede derivar en un problema social y de salud de gran importancia.

Desde el punto de vista de la sociedad en su conjunto, dichas consecuencias se ponen de manifiesto en la elevación del índice de deserción escolar especialmente femenina y las altas tasas de fecundidad adolescente.

En la actualidad, a pesar que el tema de la planificación familiar es bastante difundido, existen personas, y en especial jóvenes quienes hasta el momento no tienen acceso a información adecuada y que sumado a esto mantienen actitudes negativas que predisponen una conducta inadecuada e irresponsable, entre ellas, la maternidad precoz $(21,26,27)$.

El aborto y el embarazo a edades tempranas son problemas que se presentan por falta de información, frustrando el futuro de los jóvenes, unido a esto la grave situación económica y de desempleo que atraviesa el país $(19,20,21)$.

Las mujeres quieren, en su gran mayoria, regular su propia fecundidad. En el uso creciente de anticonceptivos en casi todos los paises en desarrollo se pone de manifiesto el deseo de las mujeres de planificar los embarazos. Muchas otras mujeres quieren espaciar o limitar los nacimientos pero no practican la anticoncepción. El gran número de abortos, con frecuencia ilegales y peligrosos también pone en relieve el deseo de las mujeres de regular su fecundidad.

El machismo, las diferencias de género, el problema de la doble moral, el dogmatismo de la Iglesia, la intensidad con la que los medios de comunicación presentan al sexo placentero, sin medir sus consecuencias, el bajo presupuesto asignado a las entidades públicas, así como la falta de personal especializado profundiza y alarga una salida al problema $(25,27)$.

\section{PROBLEMA}

¿Cuáles son las conductas, conocimientos y actitudes sexuales de los adolescentes sobre la anticoncepción?, y ¿Qué relación existe entre ellas con respecto a las variables socio-demográficas (sexo y residencia)?

\section{OBJETIVO DE LA INVESTIGACIÓN}

\section{Objetivo General:}

Identificar y comparar las Conocimientos y Actitudes Sexuales sobre Anticoncepción en estudiantes de ambos sexos que cursan el $5^{\circ}$ de Secundaria en Instituciones Educativas Estatales de la ciudad de Cusco.

\section{Objetivos Específicos:}

1. Describir las características socio-demográficas de los adolescentes.

2. Identificar las actitudes y conocimientos sexuales en función de sexo.

3. Identificar las actitudes y conocimientos sobre anticonceptivos en función de sexo.

4. Identificar los medios de información y orientación sexual.

\section{JUSTIFICACIÓN}

Postulamos que en un entorno machista, la iniciación sexual del varón es precoz y el afecto no es un intermediario. La conciencia de riesgo de embarazo o de contraer enfermedades de transmisión sexual es mínima o no existe: "a mi no me pasa nada" $(30,31,32,34)$. La salud reproductiva en nuestro país está generalmente afectada por los estereotipos de comportamiento sexual machista y patriarcal, que influyen $y$, a veces, determinan niveles de salud reproductiva realmente alarmantes, como son las tasas de embarazos no deseados, abortos, mortalidad 
materna, mortalidad infantil de hijos de madres adolescentes, entre otros $(13,14,21,25,27)$.

Dada la escasa información en cuanto al conocimiento de las actitudes y conductas de adolescentes frente a la anticoncepción en la capital y el casi inexistente conocimiento en otras regiones del país $(2,9,10,11,28,29,32)$, de ambas razones surge la necesidad de realizar el estudio con estudiantes del $5^{\circ}$ de Secundaria en la ciudad de Cusco.

La obtención de estos datos sobre actitudes y conocimientos sobre anticoncepción permitirá la comprensión y elaboración de una estrategia de intervención cognitivo-conductual que permita agilizar el cambio de actitudes en consistencia con los comportamientos mostrados.

\section{METODOLOGÍA}

\section{TIPO DE INVESTIGACIÓN}

Es una investigación de tipo exploratoria.

\section{DISEÑO DE LA INVESTIGACIÓN}

Diseño Descriptivo Simple.

\section{UNIVERSO Y MUESTRA}

La población de estudio está constituida por estudiantes que cursaban el $5^{\circ}$ año de secundaria con edades comprendidas entre 15 y 20 años, hispanohablantes, de centros educativos estatales, turno diurno, de la ciudad del Cusco.

La selección de los estudiantes, que son las unidades de análisis, se efectúo mediante una muestra aleatoria bietápica. Se seleccionaron primero la Instituciones Educativos (I.E.) y en una segunda etapa, las secciones de quinto año en las que se evaluó a todos los estudiantes.

El tamaño de la población y la muestra de las I.E. seleccionadas por ciudades se presenta a continuación:

\section{DISTRIBUCIÓN DE LA MUESTRA DE ESTUDIANTES POR CIUDAD Y SEXO}

\begin{tabular}{lcc}
\hline \multirow{2}{*}{ SEXO } & \multicolumn{2}{c}{ CUSCO } \\
\cline { 2 - 3 } & $\mathbf{N}^{\circ}$ & $\%$ \\
\hline MASCULINO & 119 & 47.2 \\
FEMENINO & 146 & 52.8 \\
\hline TOTAL & 265 & 100.0 \\
\hline
\end{tabular}

Criterios de Inclusión: Fueron considerados en el estudio los estudiantes que:

- Estén cursando el $5^{\circ}$ de secundaria y asisten en forma regular.

Residencia estable en las ciudades investigadas.

- Edad comprendida entre los 15 y 20 años.

\section{TÉCNICAS E INSTRUMENTOS DE RECOLECCIÓN DE DATOS}

\section{Instrumento:}

El Instrumento de recolección de datos comprende tres partes; información sobre conocimientos sexuales y anticoncepción, comportamientos sexuales y uso de anticonceptivos y el tercero es el Test de Actitudes. Es una encuesta anónima autoaplicable, que comprende 70 reactivos (Anexo) cuyo tiempo de aplicación es de 50 a 60 minutos.

\section{Test de Actitudes sobre la Anticoncepción:}

La escala de Actitudes consta de 25 items validados en el trabajo aplicado por Bardis, P. (8, $9,10)$ y fue adaptada para la presente investigación. El procedimiento para su construcción fue la Técnica de Likert que se conoce también como el Método de Consistencia Interna o Técnica de Suma de Puntuaciones. Cada respuesta tiene la asignación de 0 a 4, según la alternativa elegidas; como sigue:

0 . Desacuerdo total.

1. Desacuerdo.

2. Indeciso.

3. Acuerdo.

4. Acuerdo total 


\section{VALIDACIÓN DE LA ESCALA}

En el presente estudio se validó la escala mediante las correlaciones item-test.

\section{RESULTADOS}

CARACTERÍSTICAS DE LA POBLACIÓN DE ESTUDIANTES

\begin{tabular}{lcc}
\hline Ciudad & \multicolumn{2}{c}{ Cusco } \\
\cline { 2 - 3 } Sexo & $\mathrm{N}^{\circ}$ & $\%$ \\
Masculino & 119 & 44.9 \\
Femenino & 146 & 55.1 \\
\hline Total & 265 & 100.0 \\
\hline \multicolumn{1}{c}{ NIVEL EDUCATIVO DEL PADRE } \\
\hline Sin Instrucción & 1 & 0.4 \\
Primaria Incompleta & 17 & 6.4 \\
Primaria Completa & 26 & 9.8 \\
Secundaria Incompleta & 27 & 10.2 \\
Secundaria Completa & 69 & 26.0 \\
Superior Incompleta & 27 & 10.2 \\
Superior Completa & 98 & 37.0 \\
\hline Total & 265 & 100.0 \\
\hline \multicolumn{1}{c}{ NIVEL EDUCATIVO DE LA MADRE } \\
\hline Sin Instrucción & 9 & 3.4 \\
Primaria Incompleta & 37 & 14.0 \\
Primaria Completa & 23 & 8.7 \\
Secundaria Incompleta & 33 & 12.5 \\
Secundaria Completa & 69 & 26.0 \\
Superior Incompleta & 30 & 11.3 \\
Superior Completa & 64 & 24.2 \\
\hline Total & 265 & 100.0 \\
\hline
\end{tabular}

ESTADO CIVIL DE LOS PADRES

\begin{tabular}{lcc}
\hline & \multicolumn{2}{c}{ Cusco } \\
\cline { 2 - 3 } & $\mathrm{N}^{\circ}$ & $\%$ \\
\hline Unidos & 227 & 85.7 \\
Separados & 24 & 9.0 \\
Viudos & 14 & 5.3 \\
\hline Total & 265 & 100.0 \\
\hline
\end{tabular}

CON QUIEN VIVE ACTUALMENTE

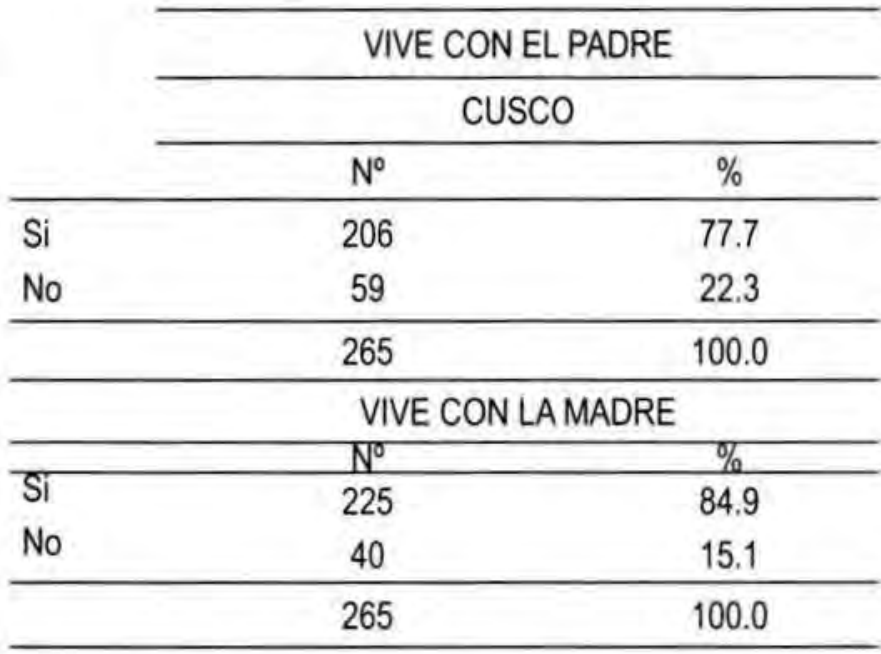

PRIMERA PERSONA QUE DIO INFORMACIÓN SOBRE ASPECTOS SEXUALES ESPONTÁNEAMENTE

\begin{tabular}{lcc}
\cline { 2 - 3 } & \multicolumn{2}{c}{ Cusco } \\
\cline { 2 - 3 } & $\mathrm{N}^{\circ}$ & $\%$ \\
\hline Padre & 71 & 26.8 \\
Madre & 87 & 32.8 \\
Familiar & 56 & 21.1 \\
Amigo(a) & 10 & 3.8 \\
Enamorado(a) & 17 & 6.4 \\
Profesional de la salud & 23 & 8.7 \\
Otras & 1 & 0.4 \\
\hline Total & $265^{\#}$ & 100.0 \\
\hline
\end{tabular}

MEDIO DE COMUNICACIÓN MASIVA MÁS CONFIABLE Y SERIO QUE DA INFORMACIÓN SOBRE ASPECTOS SEXUALES

\begin{tabular}{lcc}
\hline & \multicolumn{2}{c}{ Cusco } \\
\cline { 2 - 3 } & $\mathrm{N}^{\circ}$ & $\%$ \\
\hline Periódicos & 21 & 8.8 \\
Revistas & 28 & 11.7 \\
Radio & 58 & 24.3 \\
Televisión & 126 & 52.7 \\
Cine & 6 & 2.5 \\
\hline Total & $239^{*}$ & 100.0 \\
\hline
\end{tabular}




\begin{tabular}{|c|c|c|c|c|}
\hline \multicolumn{5}{|c|}{$\begin{array}{l}\text { A QUIÉN ACUDIRÍA PARA } \\
\text { ORIENTACIÓN SOBRE ASPECTOS } \\
\text { SEXUALES }\end{array}$} \\
\hline & \multicolumn{4}{|c|}{ Cusco } \\
\hline & \multicolumn{2}{|c|}{ Masc. } & \multicolumn{2}{|c|}{ Fem. } \\
\hline & $\mathrm{N}^{\circ}$ & $\%$ & $\mathrm{~N}^{\circ}$ & $\%$ \\
\hline Nadie & 00 & 0.00 & 00 & 0.00 \\
\hline Padre & 29 & 10.9 & 12 & 04.5 \\
\hline Madre & 14 & 05.4 & 65 & 24.5 \\
\hline Familiar & 34 & 12.8 & 27 & 10.3 \\
\hline Amigo(a) & 01 & 0.40 & 01 & 0.40 \\
\hline Enamorado(a) & 16 & 06.0 & 12 & 04.5 \\
\hline Profesional de Salud & 12 & 04.5 & 16 & 06.0 \\
\hline Religioso(a) & 00 & 0.00 & 01 & 0.40 \\
\hline Profesor(a) & 13 & 04.9 & 12 & 04.5 \\
\hline Total & 119 & 44.9 & 146 & 55.1 \\
\hline
\end{tabular}

\begin{tabular}{|c|c|c|c|}
\hline & \multicolumn{3}{|c|}{ PRIMERA MASTURBACIÓN } \\
\hline & Media & $\mathrm{N}^{\circ}$ & $\%$ \\
\hline \multirow{5}{*}{$\begin{array}{l}\text { Masculino } \\
\text { Femenino }\end{array}$} & 13.88 & $57^{\circ}$ & 89.1 \\
\hline & 11.57 & 7 & 10.9 \\
\hline & & 64 & 100.0 \\
\hline & PRIM & $\mathrm{ACI}$ & TAL \\
\hline & Media & $\mathrm{N}^{\circ}$ & $\%$ \\
\hline \multirow{3}{*}{$\begin{array}{l}\text { Masculino } \\
\text { Femenino }\end{array}$} & 13.95 & 38 . & 79.2 \\
\hline & 11.40 & $10^{*}$ & 20.8 \\
\hline & & 48 & 100.0 \\
\hline
\end{tabular}

\section{CONOCIMIENTOS Y USO DE MÉTODOS} ANTICONCEPTIVOS POR SEXO

\begin{tabular}{|c|c|c|c|c|}
\hline & \multicolumn{4}{|c|}{$\begin{array}{l}\text { QUÉ MÉTODO ANTICONCEPTIVO } \\
\text { CONOCES }\end{array}$} \\
\hline & \multicolumn{4}{|c|}{ Cusco } \\
\hline & \multicolumn{2}{|c|}{ MASCULINO } & \multicolumn{2}{|c|}{ FEMENINO } \\
\hline & $\mathrm{N}^{\circ}$ & $\%$ & $\mathrm{~N}^{\circ}$ & $\%$ \\
\hline Condón & 97 & 81.5 & 119 & 81.5 \\
\hline Píldora & 46 & 38.7 & 93 & 63.7 \\
\hline DIU & 9 & 7.7 & 30 & 20.5 \\
\hline Met. Ritmo & 28 & 23.5 & 53 & 36.3 \\
\hline Inyectab. & 24 & 20.2 & 58 & 39.7 \\
\hline Lavado V. & 18 & 15.1 & 38 & 26.0 \\
\hline \multirow[t]{5}{*}{ Coitos I. } & 10 & 8.4 & 8 & 5.5 \\
\hline & \multicolumn{2}{|c|}{$\mathrm{n}=119$} & \multicolumn{2}{|c|}{$\mathrm{n}=146$} \\
\hline & \multicolumn{4}{|c|}{$\begin{array}{l}\text { QUÉ METTODO ANTICONCEPTIVO } \\
\text { HAS USADO }\end{array}$} \\
\hline & \multicolumn{2}{|c|}{ MAscutino } & \multicolumn{2}{|c|}{ FEMENINO } \\
\hline & $\mathrm{N}^{\circ}$ & $\%$ & $\mathrm{~N}^{\circ}$ & $\%$ \\
\hline Condón & 26 & 17.6 & 5 & 3.4 \\
\hline Pildora & 2 & 1.7 & 1 & 0.7 \\
\hline DIU & 0 & 0.0 & 0 & 0.0 \\
\hline Met. Ritmo & 2 & 1.7 & 0 & 0.0 \\
\hline Inyectab. & 0 & 0.0 & 1 & 0.7 \\
\hline Lavado V. & 1 & 0.8 & 2 & 1.4 \\
\hline \multirow[t]{5}{*}{ Coitos I. } & 2 & 1.7 & 0 & 0.0 \\
\hline & \multicolumn{2}{|c|}{$\mathrm{n}=119$} & \multicolumn{2}{|c|}{$\mathrm{n}=146$} \\
\hline & \multicolumn{4}{|c|}{$\begin{array}{l}\text { QUÉ MÉTODO ANTICONCEPTIVO } \\
\text { USA ACTUALMENTE }\end{array}$} \\
\hline & \multicolumn{2}{|c|}{ MASCULINO } & \multicolumn{2}{|c|}{ FEMENINO } \\
\hline & $\mathrm{N}^{\circ}$ & $\%$ & $\mathrm{~N}^{\circ}$ & $\%$ \\
\hline Condón & 34 & 28.6 & 3 & 2.1 \\
\hline Pildora & 3 & 2.5 & 0 & 0.0 \\
\hline DIU & 0 & 0.0 & 0 & 0.0 \\
\hline Met. Ritmo & 1 & 0.8 & 0 & 0.0 \\
\hline Inyectab. & 1 & 0.8 & 1 & 0.7 \\
\hline Lavado V. & 0 & 0.0 & 2 & 1.4 \\
\hline \multirow[t]{2}{*}{ Coitos I. } & 0 & 0.0 & 0 & 0.0 \\
\hline & \multicolumn{2}{|c|}{$n=119$} & \multicolumn{2}{|c|}{$n=146$} \\
\hline
\end{tabular}

RAZÓN MÁS IMPORTANTE PARA QUE TUVIERA SU PRIMERA EXPERIENCIA SEXUAL

\begin{tabular}{lcccc}
\hline \multirow{2}{*}{\multicolumn{1}{c}{ MOTIVO }} & \multicolumn{4}{c}{ Cusco } \\
\cline { 2 - 5 } & \multicolumn{2}{c}{ Masc. } & \multicolumn{2}{c}{ Fem. } \\
\cline { 2 - 6 } $\mathrm{N}^{\circ}$ & $\%$ & $\mathrm{~N}^{\circ}$ & $\%$ \\
\hline Deseo & 01 & 0.40 & 07 & 02.6 \\
Saber lo que se siente & 62 & 23.4 & 37 & 14.0 \\
Que la mayoría lo hace & 06 & 02.3 & 12 & 04.5 \\
Diversión & 15 & 05.7 & 21 & 07.9 \\
Se casará & 13 & 04.8 & 18 & 06.8 \\
Ottos & 12 & 04.5 & 24 & 09.1 \\
Lo pidió mi pareja & 10 & 03.8 & 27 & 10.2 \\
\hline Total & 119 & 44.9 & 146 & 55.1 \\
\hline
\end{tabular}

EDAD DE LAS PRIMERAS EXPERIENCIAS SEXUALES POR SEXO

\begin{tabular}{|c|c|c|c|}
\hline & \multicolumn{3}{|c|}{ PRIMER ENAMORADO } \\
\hline & \multicolumn{3}{|c|}{ CUSCO } \\
\hline & Media & $\mathrm{N}^{\circ}$ & $\%$ \\
\hline Masculino & 13.65 & 72 & 47.7 \\
\hline Femenino & 14.64 & 79 & 52.3 \\
\hline & & 151 & 100.0 \\
\hline
\end{tabular}


CONOCIMIENTO SOBRE LA

PROTECCION Y SEGURIDAD

QUE BRINDAN LOS METODOS ANTICONCEPTIVOS SEGÚN SEXO

\begin{tabular}{lcccc} 
& \multicolumn{3}{c}{$\begin{array}{c}\text { QUÉ MÉTODO ANTICONCEPTIVO } \\
\text { PROTEGE MÁS DEL SIDA }\end{array}$} \\
\cline { 2 - 5 } & \multicolumn{4}{c}{ CUSCO } \\
\cline { 2 - 5 } & \multicolumn{3}{c}{ MASCULINO } & \multicolumn{3}{c}{ FEMENINO } \\
\cline { 2 - 5 } & $\mathrm{N}^{\text {o }}$ & $\%$ & $\mathrm{~N}^{\circ}$ & $\%$ \\
\hline Condón & 76 & 63.9 & 84 & 57.5 \\
Píldora & 5 & 4.2 & 5 & 3.4 \\
DIU & 4 & 3.4 & 2 & 1.4 \\
Met. Ritmo & 1 & 0.8 & 3 & 2.1 \\
Inyectable. & 3 & 2.5 & 5 & 3.4 \\
Lavado V. & 3 & 2.5 & 4 & 2.7 \\
Coitos I. & 1 & 0.8 & 4 & 2.7 \\
\hline
\end{tabular}

$\mathrm{n}=119 \quad \mathrm{n}=146$

QUÉ MÉTODO ANTICONCEPTIVO

ES MÁS SEGURO

\begin{tabular}{|c|c|c|c|c|}
\hline & \multicolumn{4}{|c|}{ CUSCO } \\
\hline & \multicolumn{2}{|c|}{ MASCULINO } & \multicolumn{2}{|c|}{ FEMENINO } \\
\hline & $\mathrm{N}^{\circ}$ & $\%$ & $\mathrm{~N}^{\circ}$ & $\%$ \\
\hline Condón & 56 & 47.1 & 58 & 39.7 \\
\hline Píldora & 10 & 8.4 & 17 & 11.6 \\
\hline DIU & 3 & 2.5 & 9 & 6.2 \\
\hline Met. Ritmo & 7 & 5.9 & 7 & 4.8 \\
\hline Inyectab. & 5 & 4.2 & 11 & 7.5 \\
\hline L. Vaginal & 5 & 4.2 & 0 & 0.0 \\
\hline \multirow[t]{2}{*}{ Coitos I. } & 2 & 1.7 & 3 & 2.1 \\
\hline & \multicolumn{2}{|c|}{$\mathrm{n}=119$} & \multicolumn{2}{|c|}{$\mathrm{n}=146$} \\
\hline
\end{tabular}

QUÉ MÉTODO ANTICONCEPTIVO ES EL QUE MÁS USAN LOS JÓVENES SEGÚN SEXO

\begin{tabular}{lcccc}
\cline { 2 - 5 } & \multicolumn{4}{c}{ CUSCO } \\
\cline { 2 - 5 } & \multicolumn{2}{c}{ MASCULINO } & \multicolumn{2}{c}{ FEMENINO } \\
\cline { 2 - 5 } & $\mathrm{N}^{\circ}$ & $\%$ & $\mathrm{~N}^{\circ}$ & $\%$ \\
\hline No respondieron & 16 & 13.4 & 24 & 16.4 \\
Condón & 101 & 84.9 & 117 & 80.2 \\
Pildora & 2 & 1.7 & 5 & 3.4 \\
DIU & 0 & 0.0 & 0 & 0.0 \\
Ritmo & 0 & 0.0 & 0 & 0.0 \\
\hline & \multicolumn{5}{c}{$\mathrm{n}=119$} & $\mathrm{n}=146$ \\
\cline { 2 - 5 }
\end{tabular}

MEDIA DEL PUNTAJE TOTAL EN LA ESCALA DE ACTITUDES POR SEXO

\begin{tabular}{lccc}
\hline & MEDIA & DEsviACiÓN STANDARD & $\mathrm{N}^{\circ}$ \\
\cline { 2 - 4 } Masculino & 56.64 & 13.20 & 119 \\
Femenino & 58.29 & 15.02 & 146 \\
\hline
\end{tabular}

\section{DISCUSIÓN}

La discusión se centrará fundamentalmente en el análisis de los hallazgos que son referentes a los objetivos de la presente investigación, para ello se iniciará con las características demográficas de la población, luego se analizará los resultados sobre información y experiencias sexuales, para posteriormente culminar con el estudio sobre conductas y actitudes frente a la anticoncepción.

\section{Características Poblacionales}

De la población total correspondiente a la muestra de la ciudad del Cusco, en promedio, el $77 \%$ de los encuestados señala vivir con el padre, el $84.9 \%$ vivir con la madre. No se tienen datos al respecto, pero los encontrados coinciden con los encontrados por Chirinos, J. $(1,996)$ y La Rosa, L. $(1,995)$ y Salazar, H. $(1,997)$.

De los datos se puede inferir la reducción del espacio vital, tendencia al hacinamiento, violencia doméstica y dificultad en la satisfacción de necesidades básicas, que se hace más álgidas y evidentes en zonas urbano-marginales y que antes era casi exclusiva de Lima y que conlleva la necesidad a que ambos padres trabajen o inclusive lo hagan los hijos.

Lo anterior contribuiria en la disminución de la comunicación positiva, de tipo afectivo-verbal y que los hogares queden sin la supervisión requerida para la educación de los hijos con consecuencias de bajo rendimiento y/o deserción escolar, y/o que hermanos menores asuman roles que no son coherentes para su edad, con los riesgos que esto implica, como lo señala la comunicación $\mathrm{N}^{\mathrm{a}} 6$ de la OPS que trata sobre la "Salud de los adolescentes y los jóvenes de América" (21). De los hallazgos anteriores se puede señalar que entre el $8.7 \%$ a 
$14.3 \%$ viven con uno de suspadres $y /$ o con los familiares de ellos, y el $23 \%$ de ellos son padres separados o que nunca convivieron, lo que significa que de alguna manera es una población de adolescentes en situación de alto riesgo, con mayor probabilidad de desarrollo deficitario y $\sin$ modelos psicosexuales adecuados.

Respecto al NIVEL EDUCATIVO DE LOS PADRES de lo informado por los estudiantes, en promedio el $55 \%$ de los progenitores tienen entre Secundaria Completa y Educación Superior Completa predominando el nivel de educación de los padres $(63 \%)$ en comparación con las madres $(50 \%)$, las diferencias se particularizan en el hallazgo de que $37 \%$ de los padres tienen Educación Superior vs. $24.2 \%$ de las madres.

Los hallazgos reflejan el mantenimiento de la valoración psicosocial que se asigna al varón y que se traducen en oportunidades educativas, laborales y económicas.

\section{Información Sexual:}

Respecto a la PERSONA DE MAYOR CONFIANZA para hablar DE "problemas personales" es la madre a quien señalan en primer lugar $(24 \%)$ seguido por un familiar $(10 \%)$ y el padre $(4.5 \%)$. Los resultados reflejan la importancia afectiva que tiene la progenitora y la trascendencia que adquiere en su función orientadora; es probable que este rol de confidente lo adquiere por el papel socio-familiar que el sistema social tradicionalmente le adjudica a la mujer (pasivo-afectivo, tolerante, comprensiva, sumisa, etc.) y al hombre le encarga cumplir como padre el rol de "autoridad" (agresivo, práctico, poco afectivo), esto explicaría por qué eligen a un familiar como segundo confidente. Los resultados no coinciden con los encontrados en las investigaciones de Chirinos, J. (1,996), La Rosa, L. (1,995), Gonzáles, G. y col. $(1,994)$ que concluyen que son los amigos a los que los adolescentes señalan como la persona de mayor confianza para conversar sobre sus problemas sexuales. Habría que investigar la diferencia entre comentar, compartir y consultar o buscar consejo respecto a un problema sexual.

En referencia a los MEdios DE comunicación, señalan a los Periódicos como el que brinda mayor información sobre aspectos sexuales $(37 \%)$, sin embargo es la información que brinda la Televisión a la que indican como la más confiable $(52.7 \%)$. Es de importancia señalar que es a la Radio a quien señalan en segundo lugar como la más confiable ( $24 \%$ ); al respecto es importante considerar el valor de la radio como medio de información y educación no solamente sexual por su gran alcance social y geográfico a muy bajo costo en comparación con la Televisión. Tanto la Radio como la Televisión deberían estar comprometidas en proyectos nacionales de educación que incluyan programas preventivos sobre aspectos sexuales y en la modificación de costumbres y actitudes negativas que comprometen la salud sexual. No se debe olvidar que en los hogares hay un gran consumo de tiempo en escuchar Radio y ver Televisión, fundamentalmente cuando ambos progenitores trabajan son los niños los primeros consumidores, por lo tanto las programaciones deberían estar constantemente supervisadas por los sectores estatales respectivos.

\section{EXPERIENCIAS SEXUALES}

El $47 \%$ de los estudiantes varones y el $52 \%$ de mujeres han tenido enamorado(a) y un promedio del $50 \%$ no lo tienen, este hallazgo difiere en algo con lo encontrado por Chirinos, J. $(1,996)$, La Rosa, L. $(1,995)$ y Soto, V. $(1,994)$.

$\mathrm{Al}$ respecto la reflexión de Erickson señala que la "Etapa de enamoramiento no es en modo alguno total o siquiera primariamente sexual, salvo cuando las costumbres lo exigen. $\mathrm{El}$ amor del adolescente constituye un intento por llegar a una definición de la propia identidad. A esto se debe que una parte tan considerable del amor adolescente consista en conversación"; el $60 \%$ 
que no tiene actualmente enamorado puede ser un indicador psicosocial que evidencia los resultados de la coeducación o la presencia de hechos sociales que están ocupando o compensando esta necesidad $(8,9,18,32,34)$.

El $89 \%$ de los varones encuestados y el $10.69 \%$ de las mujeres se ha masturbado, esta diferencia significativa por sexos reflejaría en parte la fuerza de la necesidad psicobiológica del varón que se satisface con este tipo de experiencia. Ya sea la presión social, la curiosidad o el placer, esta experiencia sería la forma de satisfacer impulsos y de disminuir riesgos y consecuencias negativas en la salud sexual. De hecho estos resultados, aparte de que es un indicador temprano de la característica de expresión social de la sexualidad, debe tomarse en cuenta para dirigir programas preventivos, de intervención temprana focalizados en los adolescentes varones.

Las experiencias coitales encontradas en estudiantes varones es de $79 \%$ y en las mujeres es $20.8 \%$, siendo el(la) enamorado(a) la primera pareja coital, y como razón fundamental "saber que se siente" (varones 23\% y mujeres 14\%); el haber encontrado como edad promedio de esta experiencia, es a los 14 para los varones y 11 para las mujeres, nos señalan las características de un marco social donde la prevalencia de iniciación sexual es mayor para los varones y que si bien esta diferencia puede estar disminuyendo a través del tiempo, se mantienen los roles sexuales estereotipados en los que la sociedad otorga al varón el "rol de iniciativa y responsabilidad sexual". Estos hallazgos son similares a los encontrados por Alarcón, I. (1,992); Soto, V. $(1,994)$ y La Rosa, L. $(1,995)$.

\section{ANTICONCEPCIÓN}

Encuantoalusode métodos anticonceptivos, el $17.6 \%$ de los varones y el $3.4 \%$ de las mujeres, afirman haberlos usado; señalando al condón $(81 \%)$ y a la píldora $(50 \%)$ como los más conocidos. Si hacemos la comparación de porcentajes entre conocimientos de uso de anticonceptivos y de experiencia coital según sexo encontramos que en los varones hay una gran desproporción, que configuraría un claro desconocimiento que llega a la irresponsabilidad, con consecuencias que esto conlleva, ubicándole en una situación de alto riesgo (Cáceres y col 1,992, OPS 1,995; Alarcón, I. 1,992; Loli 1,986).

Lo anterior se hace más emergente o muy preocupante cuando se encuentra como resultado entre un $2 \%$ a $4 \%$ de la población investigada que señalan a la píldora, DIU, lavado vaginal, inyectables y coitos interruptus como métodos que protegen del SIDA, esto se hace más grave aún si agregamos el porcentaje de la población que no contestó por no saberlo o por inseguridad, cuyo resultado se obtendría de la diferencia entre el $60 \%$ en promedio que respondieron que el CONDÓN protege contra el SIDA, y el $2 \%$ a $4 \%$ que responden señalando esta acción a los otros métodos anticonceptivos, lo que daría un $26 \%$ adicional de población en riesgo.

En opinión de los estudiantes varones y mujeres el Condón es el anticonceptivo más usado $(80 \%)$ señalando como razones para ello primero porque "es más seguro" segundo porque "es más barato" y tercero porque es "fácil de encontrar". En cuanto a las razones personales que impiden el uso de anticonceptivos señalan en primer lugar la "falta de información ", luego la "vergüenza para comprarlo" y finalmente "el ser descubierto".

Los resultados encontrados nos llevan a identificar la disonancia cognitivo-conductual de efectos potencialmente graves, dado que se encuentra, por un lado, desconocimiento de los métodos anticonceptivos, aunque un alto porcentaje dice "conocerlos" y, por otro lado, se encontró respecto al ejercicio coital un alto porcentaje de los estudiantes que reconocía barreras por las que no pueden usar métodos anticonceptivos (falta de información $64 \%$ ).

La disonancia puede ser resuelta, porque 
cuando esta misma población reconoce que la responsabilidad para el uso de anticonceptivos debe ser asumida "por la pareja" y opina mayoritariamente que sobre ello debe enseñarse desde el $3^{\circ}$ grado de secundaria, esto facilita y marca la oportunidad para intervenciones psicoeducativas. Los resultados coinciden con lo hallado por Chirinos, J. $(1,996)$; La Rosa, L. $(1,995)$ y Zambrano, D. $(1,994)$.

\section{ACTITUDES}

Los resultados que se encontraron con respecto a las actitudes frente a la anticoncepción, de los estudiantes es predominantemente Semiliberal $(66.2 \%)$ seguido por una actitud Semiconservadora $(26.9 \%)$ sin diferencias significativas por sexo. Aunque esta relación no sea significativa estadísticamente es importante como variable de influencia.

$\mathrm{Si}$ bien es cierto, predomina la actitud Semiliberal, que implica un reconocimiento de la necesidad de planificar el número de hijos y una mayor probabilidad de aceptación de los anticonceptivos como medio de lograrlo, es necesario reconocer que las barreras para el logro de este objetivo radican en la falta de información sobre sus efectos, uso correcto y limitaciones de cada método y por otro lado en la persistencia, aunque menor, de la inconsistencia entre la actitud, conocimiento y comportamiento sexual ( $90 \%$ señala de 19 a 20 años la edad para usar anticonceptivos).

Las inconsistencias se encuentran en la opinión sobre las edades, que señalan los encuestados, para diferentes experiencias afectivosexuales como son para tener enamorado(a) (15 años), para la primera experiencia coital (19.5 años) o sobre el conocimiento sobre anticonceptivos y lo que dicen "conocer" y lo que hacen en realidad.

En cuanto a la instrucción de los padres y la actitud es cierto que no se encuentra relación estadística significativa, sin embargo, se encuentra la tendencia que a mayor instrucción de los progenitores la actitud tiende hacia la liberalidad, tendencia que de alguna manera podría también relacionarse con el acceso a programas de T.V. o a la lectura de revistas o periódicos como fuentes de información, esta debería ser motivo de un estudio más profundo.

Para finalizar es bueno reflexionar señalando que el éxito con que los adolescentes integren la sexualidad en el marco total de sus vidas depende de una serie compleja de factores que van desde la calidad de la interacción con sus padres, hasta la influencia de la aparición de nuevas normas morales y sociales. Estas nuevas normas han producido por una parte una liberación de prejuicios de larga data, relacionados con la sexualidad, pero, por otra parte, han conducido a un aumento de los problemas que surgen de la liberación de la conducta sexual (OPS N ${ }^{\circ}$ 6-1,995).

\section{CONCLUSIONES}

1. El mayor porcentaje de estudiantes encuestados vive con ambos progenitores, cuyo grado de instrucción se ubica entre la educación secundaria a la superior, encontrándose que los padres tienen cierta ventaja en el nivel educativo en comparación con las madres.

2. La familia es el centro de información sexual, es la madre quien cumple un rol afectivo y de orientación seguido por el padre y el(la) amigo(a).

3. Los estudiantes señalan entre las fuentes de comunicación masiva que le proporcionaron información sobre sexualidad; primero al periódico, luego a las revistas y la radio/T.V;; sin embargo, es a la T,V. a las que señalan como la más confiable y seria.

4. S on los varones (en mayor proporción que las mujeres) quienes tuvieron experiencia coital. La edad promedio para ambos sexos es entre los 14 y 15 años y la pareja de la primera experiencia ha sido el(la) enamorado(a).

5. El condón es el anticonceptivo más conocido y usado, seguido por la píldora. Consideran que el 
condón es el más seguro, barato y más accesible que protege del SIDA.

6. Elconocimiento sobre sexualidad, reproducción $\mathrm{y}$ anticoncepción es bajo, y existe disonancia entre las actitudes, conocimiento y experiencia sexual.

7. La actitud frente a la anticoncepción de la muestra en la ciudad del Cusco es semiliberal.

\section{REFERENCIAS BIBLIOGRAFICAS}

Alarcón, 1. Comportamiento Sexual en Adolescentes de Colegios Estatales de Lima, Cusco e Iquitos. Instituto de Investigaciones de Altura. UPCH, 1,992.

Arias, J. Estudio de Actitudes Frente a la Sexualidad en Estudiantes de Medicina de la Universidad Peruana Cayetano Heredia. Tesis para optar el grado de Bachiller en Medicina, Lima, 1,976.

Bardis, P. Marriage and Family Living. 1,959.

Bardis, P. A Technique for the Measurement of Attitudes Toward Enligion. Noviembre 1,961.

Bardis, P. A Dating Scale: New York: McGraw Hill. $1,967$.

Bardis, P. Escala para la Pildora. Social Science, Enero 1,969.

Cáceres, Rosasco, Mandel y Hearts. Evaluación de una Intervención Preventiva de ETS/SIDA en el ambito escolar en el Perri. Journal of Adolescent Health. Noviembre. 1,967.

Chirinos, J. Niveles de Conocimientos, Actitudes y Prácticas Asociadas al Comportamiento Sexual de Riesgo en Escolares Adolescentes de dos Colegios de la USE 06, Lima - Norte. Tesis Doctoral en Salud Pública. UPCH, Perú. 1,996.

Egusquiza, O. Actitudes y Conocimientos Sexuales en Alumnos de Ambos Sexos del $5^{\circ}$ año de Secundaria del Cono Norte de Lima. Tesis Bach. Psic. UPCH. Lima. 1,982.

Euribe, C. Nivel de Conocimientos Sobre la Sexwalidad Humana de los Graduados de Medicina de la Ciudad de Lima. Tesis Bach. UPCH. Lima. 1,975 .
Ghilino, P. Investigación Exploratoria sobre el Nivel de Conocimiento y Actitudes hacia la Sexualidad Humana en Estudiantes de Psicologia de la Pontificia Universidad Católica y la Universidad Nacional Mayor de San Marcos. Tesis Bach. P.U.C. Lima. 1,977.

Gonzáles, G. La Adolescencia en el Perú. Instituto de Investigaciones de Altura. UPCH. Programa de Reproducción Humana. OMS. 1,994.

I.N.E.I. Estudio de los Niveles de Vida en el Perú 1991. 1992. Lima. 1,993.

I.N.E.I. Peri: Estadisticas de los Niños y Adolescentes (Compendio 1992-93). Lima. 1,994.

Kirby, D., Waszak, C., and Ziegler, J. Six schookbased clinics: Their reproductive bealth services and impact on sexual bebavior: Family Planning Perspectives 23(1): 6-16. Jan.-Feb. 1,991.

Krench, D. and Crutchfield, R. Individual in Society. New York: Mc Graw Hill. 1,962.

La Rosa, L. La Salud de los Adolescentes y Jóvenes en el Perú. Centro de Salud Pública - UPCH. Lima. 1,993.

La Rosa, L. Iniciación Sexual de los Estudiantes de $3^{\circ}$ a $5^{\circ}$ de Instrucción Secundaria de Centros Educativos del cono Sur de Lima. Tes. Maestría UPCH. Lima. 1,995.

Loli, A. La Sexualidad en las Gestantes Adolescentes. SMMISA. Lima. 1,985-1,986.

Marín, C. Anticoncepción de Elección para los Jóvenes. Niños. $\mathrm{N}^{\circ}$ 6. May. Lima 1,989.

O.P.S. La Salud de los Adolescentes y Jóvenes en las Américas. Comunicación para la Salud $\mathrm{N}^{\circ}$ 6. Washington. 1,995.

Populations Council. Manual de Encuestas de Fecundidady Planificación Familiar (Conocimientos, Actitud y Práctica). Ed. P.C. New York. 1,973. Populations Reports. Condones. Serie H, No 8 - Sep. Vol. 18 - No 3. Maryland. 1,990.

Populations Reports. Control de las Enfermedades de Transmisión Sexual. Serie L, No 9. Vol. 21 - No 1. Maryland. 1,993.

Populations Reports. Oportunidades para la Mujer Mediante la Libre Decisión Reproductiva. 
Serie M, No 12. Vol. 22 - $\mathrm{N}^{\circ}$ 1. Maryland. $1,994$.

Populations Reports. Programas de Planificación Familiar: Serie J, $\mathrm{N}^{\circ}$ 40. Maryland. 1,994.

PROMUDEH. Las Mujeres en el Perí. Lima. 2,001.

Salazar, H y Díaz, A. Conocimientos y Actitudes Sexuales. UPCH. Perú. 1,977.

Salazar, H. y Díaz, A. Cuestionario sobre Conocimientos y Actitudes Sexuales para Alumnos del $5^{\circ}$ Año de Secundaria. UPCH. Lima. 1,977.

Salazar, H. La Enseñanza de la Sexwalidad en las Facultades de Psicología de América Latina. Ponencia en el Congreso Iberoamericano de Psicología. Colegio Oficial de Psicólogos de España y la Sociedad Interamericana de Psicología. Madrid. Julio. 1,992.

Salazar, H. Salud Sexual. Boletín de Psicologia. UPCH No 3 pp 7-10. Lima. 1,995.

Salazar, H. "Conductas y Actitudes de Estudiantes Adolescentes en Relación a los Métodos Anticonceptivos" Estudio realizado en alumnos del $5^{\circ}$ de Secundaria de los Distritos de los Olivos y San Martín de Porres en Lima. Tesis Doctoral Salud Pública. Universidad Peruana Cayetano Heredia. Lima. 1,997.

Sánchez, J.; Gotuzzo, E.; Aramburú et al. Evaluación del Comportamiento Sexual en Escolares de Secundaria en la Ciudad de Yurimaguas. Resúmenes I congreso Peruano de Enfermedades Infecciosas y Tropicales. 1,989 .

Soto Cáceres, V. Comportamiento Sexual del Universitario Peruano. Chiclayo. Edit. Chiclayo SRL. 1,989.

Vega-Centeno, I. Amory Sexualidad en los Tiempos de SIDA. Ministerio de Salud, PECOS. Lima. $1,994$.

Zambrano G., D.J. Construcción de un Cuestionario de Conocimientos de Actitudes hacia los Métodos Anticonceptivos en Escolares de $5^{\circ}$ Año de Instrucción Secundaria de Colegios Estatales del Distrito de la Victoria. Tesis Bach. U.P.C.H. Lima. 1,995. 